\title{
Diagnostic Application of Fractal Dimension Analysis of Bone Invasion in Oral Malignancy-A Preliminary Retrospective Study
}

\author{
Archana $\mathrm{M}^{1}$ Jayachandran Sadaksharam ${ }^{1}$ \\ ${ }^{1}$ Department of Oral Medicine and Radiology, Tamil Nadu \\ Government Dental College and Hospital, Chennai, India
}

\begin{abstract}
Address for correspondence Archana M, BDS, Department of Oral Medicine and Radiology, Tamil Nadu Government Dental College and Hospital, Muthusamy Salai, Chennai, Tamil Nadu 600003, India (e-mail: archanam.95@gmail.com).
\end{abstract}

\begin{abstract}
Keywords

- fractal dimension analysis

- panoramic radiography

- bone invasion

- oral malignancy

Introduction Fractal dimension (FD) analysis gives a numerical measure of the degree of boundary irregularity or surface roughness of an object and is based on quantitative analysis of features in an image. It quantifies the trabecular pattern of bone by analyzing the trabecular bone and bone marrow detecting the early changes in alveolar bone mineral content. The aim of this study is to assess the alveolar bone density by FD analysis in digital orthopantomograms (OPGs) showing bony erosion. Materials and Methods The OPGs of 10 patients from the archives of Department of Oral Medicine and Radiology were included in this retrospective study. The radiographs were selected based on set inclusion and exclusion criteria. FD analysis through box counting (using Image] software) was applied to the digital radiographs to establish a noninvasive evaluation of bone structure. The FD values obtained were recorded. Results The FD values were recorded and the data were analyzed. FD values showed statistical significance with $p<0.05$.

Conclusion With the number of samples analyzed and the results obtained, it can be concluded that FD analysis is an innovative method which can be used for early diagnosis of bony invasion in oral cancer. It can serve as a guide to dental surgeons in identifying the extent of bony invasion and can help in determining the margins of surgical resection of oral malignancy. This will prove to be a useful tool in surgical planning of oral malignancy.
\end{abstract}

\section{Introduction}

Fractal geometry was introduced by the French American mathematician B. B. Mandelbrot in 1977. The term "fractal" is derived from the Latin adjective "fractus" which means broken. Fractal analysis (FA) is a method for describing complex shapes and structural patterns and expressed numerically as fractal dimension (FD). ${ }^{1}$ It gives a numerical measure of the degree of boundary irregularity or surface roughness of an object and is based on quantitative analysis of features in an image. The most popular method used for FD analysis is the box-counting method. Panoramic radiography is highlighted in this context because it is frequently taken in the dental office; it is rapid and low cost and uses low dose of radiation. ${ }^{2}$
With recent advances in digital technology, image analysis has become an essential part of several research areas from nanotechnology to neuroscience. ${ }^{3} \mathrm{FD}$ analysis using digital orthopantomograms (OPGs) is a novel method, which can be used for early diagnosis of bony invasion by oral cancer.

The aim of the study was to assess the alveolar bone density by FD analysis in OPGs showing bony erosion.

\section{Materials and Methods}

The OPGs of 10 patients ([ $n=10]$ : 5 posterior mandibles and 5 posterior maxillae) from the archives of Department of Oral Medicine and Radiology of our college were included in this 
retrospective study. The radiographs were selected from the digital OPGs taken from June to December 2019 based on the below-mentioned inclusion and exclusion criteria. The permission was taken from the head of the department before accessing the digital archives of the radiographs. OPGs showing bony erosion involving the mandible and maxilla were included in the study. OPGs with artifacts and poor-quality radiographs, radiographs with bony erosion involving the maxillary antrum, any other obvious bony pathology, and surgical reconstruction or fixation plates were excluded from the study.

All the digital OPGs were retrieved from the Sidexis XG software and were stored as JPEG files. A rectangular region of interest (ROI) was drawn on the normal side and the side

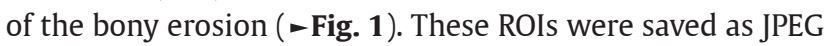
image files.

Image version 1.4.3 software was used to analyze the 20 ROIs. The ROI was opened with the File option of Image J software, and converted into 8-bit binary image ( - Fig. 1). FD value was calculated using box-counting method by selecting the Analyze option. This generates a plot containing FD value ( $\boldsymbol{- \text { Fig. }}$ 2). The FD values obtained were analyzed using the Statistical Package for Social Sciences (SPSS) Statistics, version 20 (IBM SPSS Statistics, Armonk, New York, United States).

\section{Results}

The mean FD values were found to be 1.802 and 1.742 for the unaffected side and affected side (side of the bony erosion), respectively. The statistical test used was one sample $t$-test. The standard deviation was 0.057169 and 0.075896 for the unaffected side and affected side, respectively. The standard error of mean was 0.018078 and 0.024000 for the unaffected side and affected side, respectively ( - Table $\mathbf{1}$ ).

FD values of both sides were compared ( - Fig. $\mathbf{3}$ ). The values showed statistical significance with $p<0.05$ ( - Table 2).

\section{Discussion}

By innovative applications of digital dental images, it is now feasible to improve diagnosis by image enhancement and quantitative analysis of features in an image. ${ }^{4}$ This facilitates not only early diagnosis of some diseases but also a more accurate estimation of extent of disease and its prognosis. In our study, the image analysis software employed was ImageJ software which is a Java-based image processing program developed at the National Institute of Health (Bethesda, Maryland, United States) designed for scientific multidimensional images. It can perform a wide variety of imaging manipulations with applications ranging from skin analysis to neuroscience. ${ }^{3}$

In the present study, an inexpensive and readily available method, that is, FD analysis via box counting, was applied to OPGs to establish a noninvasive evaluation of their bone structures, facilitating early diagnosis of bone invasion by oral cancer.

FD analysis has earlier been used in the diagnosis of bone diseases such as hyperparathyroidism, hemolytic anemia, implant site assessment, and postendodontic and orthodontic bone changes. It gained high importance as a diagnostic tool because of its reproducibility, reliability, widespread availability, noninvasiveness, and being cost-effective. ${ }^{4,5}$ In the present study, we have applied the principle of FD analysis for bone changes due to oral malignancy in mandible and maxilla.

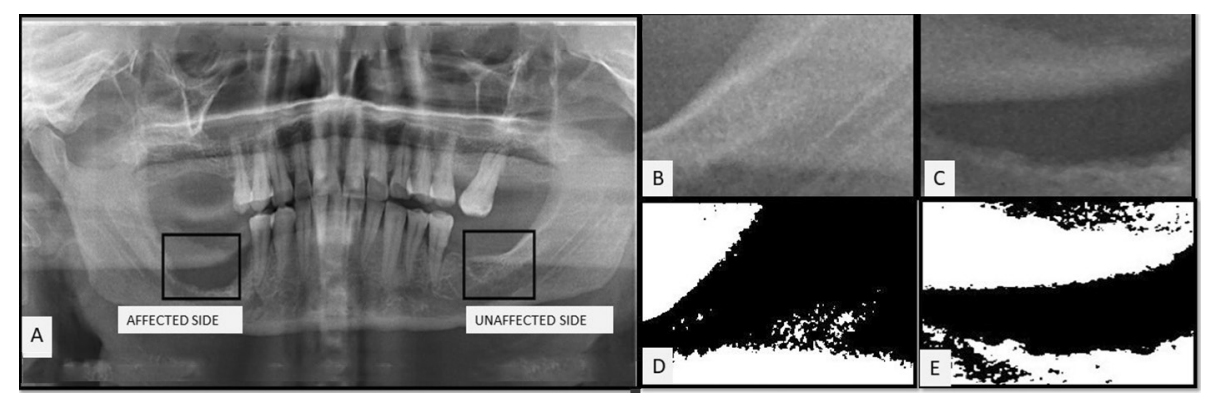

Fig. 1 (A) Digital orthopantomogram showing the affected side and the unaffected side. Region of interest: (B) unaffected side and (C) affected side. Conversion to 8-bit binary image: (D) unaffected side and (E) affected side.

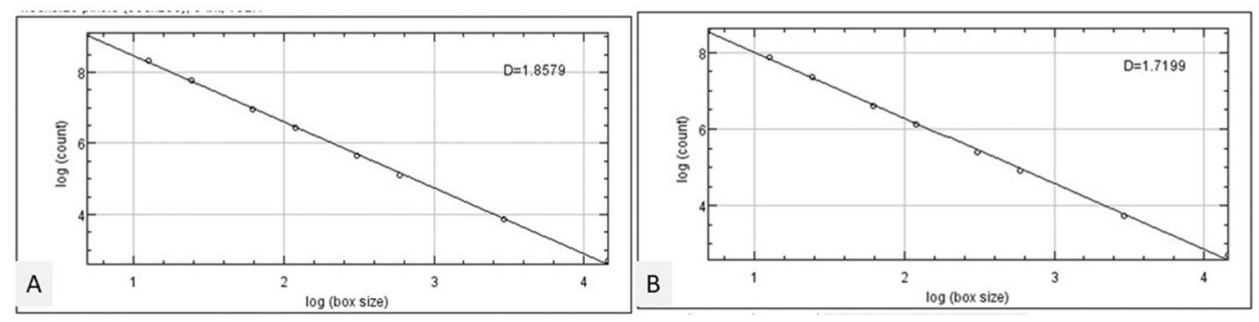

Fig. 2 Plot showing the fractal dimension value. (A) Unaffected side and (B) affected side. 
Table 1 One sample statistics

\begin{tabular}{|l|l|l|l|l|}
\hline & $\boldsymbol{n}$ & Mean & Standard deviation & Standard error of mean \\
\hline Unaffected side & 10 & 1.80250 & 0.057169 & 0.018078 \\
\hline Affected side (site of bony erosion) & 10 & 1.74230 & 0.075896 & 0.024000 \\
\hline
\end{tabular}

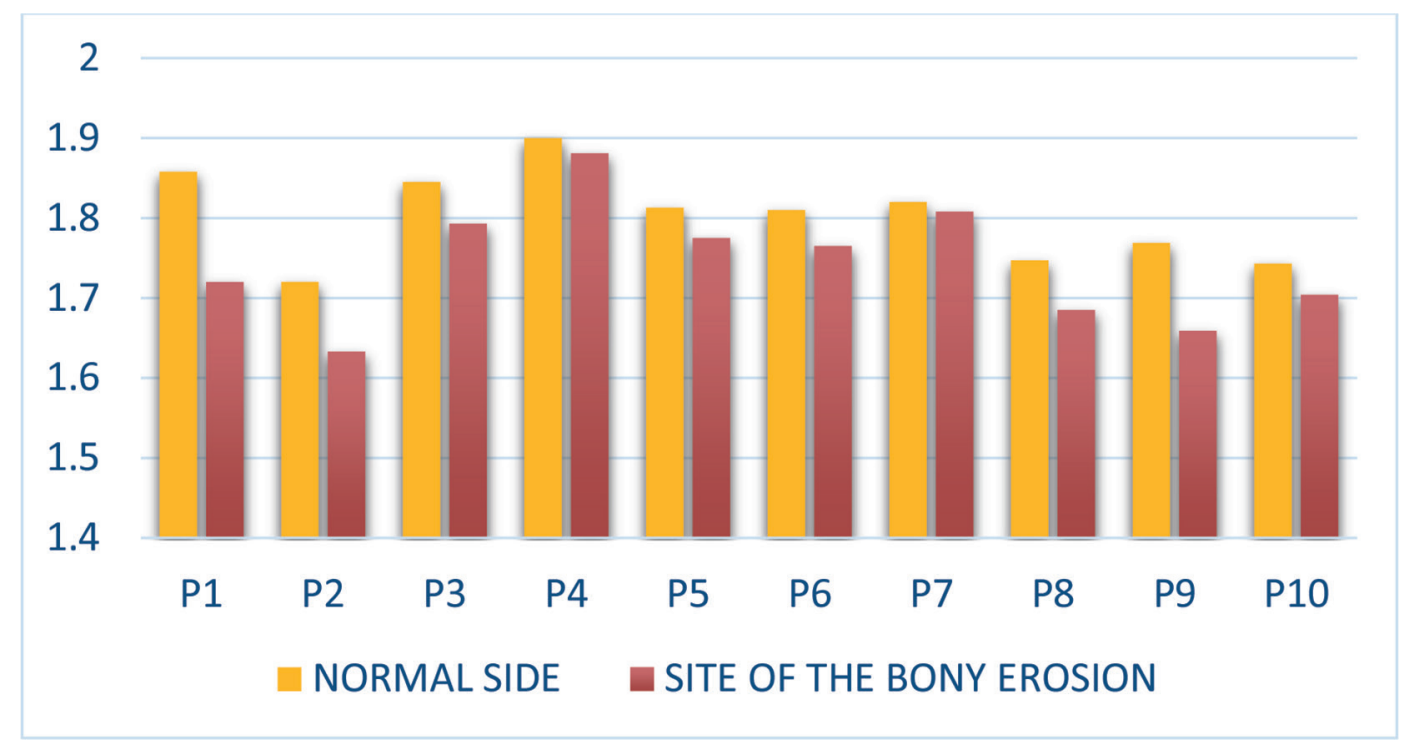

Fig. 3 Comparison of FD values between the normal side and the site of bony erosion. FD, fractal dimension.

Table 2 Comparison between unaffected side and affected side (site of bony erosion) by one sample $t$-test

\begin{tabular}{|c|c|c|c|c|}
\hline & \multicolumn{4}{|c|}{ Test value $=0$} \\
\hline & \multirow[t]{2}{*}{$t$} & \multirow{2}{*}{$\begin{array}{l}\text { Significance } \\
\text { (two-tailed) }\end{array}$} & \multicolumn{2}{|c|}{$95 \%$ confidence interval of the difference } \\
\hline & & & Lower & Upper \\
\hline Unaffected side & 99.705 & $<0.05$ & 1.76160 & 1.84340 \\
\hline Affected side (site of bony erosion) & 72.594 & $<0.05$ & 1.68801 & 1.79659 \\
\hline
\end{tabular}

The results of our study showed FD values between 1.72 and 1.90 in the normal side and between 1.63 and 1.88 on the side of the bony erosion. The results of FD values were significant in our study. The present study found that using FA, changes in the bone density can be detected at the very early stages, that is, it can be detected before $40 \%$ demineralization of bone occurs for it to be detected radiologically.

In FA, a box-counting algorithm is mainly used to quantify the trabecular pattern by counting the trabecular bone and bone marrow interfaces. ${ }^{6}$ The trabecular bone has a branching pattern that exhibits fractal properties such as self-similarity and lack of well-defined scale. Because of this phenomenon, the application of fractal geometry and the measurement of FDs can be used to determine trabecular complexity and bone structure. .,6 $^{5}$

A higher FD value indicates a more complex structure and in bony erosion, there is a change toward decreased complexity of bone and a decreased FD value, which directly relates to the reduction in the bony trabeculae. This is in agreement with White and Rudolph, Tosoni et al, and Lee and White, who in their study stated that radiographic variables that are associated with osteoporosis showed a change toward decreased complexity of the bone, thereby decreasing FD values. $^{7-9}$ Similarly, a study by Southard et al substantiated that radiographic intensity surface becomes smoother (less complex) in patients with osteoporosis, thereby decreasing the FD values. ${ }^{10}$

Previous studies have used this technique to analyze osteoporotic bone changes in iliac crests, axial bones, and tumors with promising results.

Quantitative computed tomography (CT) or dual photon X-ray absorptiometry could be used to measure the bone density but in the clinical circumstance of the dental clinic, their usefulness is limited due to the lack of proper equipment and the difficulty of carrying out the procedures. ${ }^{11,12}$ It is also possible to measure bone density in the ROI with the Hounsfield unit using conventional CT. In spite of these various options, most clinicians still prefer panoramic radiographic imaging for evaluating bone quality. ${ }^{13}$

This simple, inexpensive, and noninvasive method can prove to be an effective screening tool for detection of bone changes before $40 \%$ demineralization has taken place. Electronic archiving of data is possible as it is done on digital 
radiographs. The limitation of this study is the requirement of digital radiographs.

\section{Conclusion}

With the results obtained from the present study, it can be concluded that FD analysis is an innovative method which can be used in cases of bony invasion in oral malignancy. It can serve as a guide to dental surgeons in identifying the extent of bony invasion and can help in determining the margins of surgical resection of oral malignancy. This will prove to be a useful tool in surgical planning of oral malignancy. Validation with a larger population of varied ethnic diversity is recommended for wider applicability and generalization of the results of this study. This study will be extended in a prospective manner with a larger sample size.

\section{Conflict of Interest}

None declared.

\section{References}

1 Toghyani S, Nasseh I, Aoun G, Noujeim M. Effect of image resolution and compression on fractal analysis of the periapical bone. Acta Inform Med 2019;27(3):167-170

2 Camargo AJ, Cortes ARG, Aoki EM, Baladi MG, Arita ES, Watanabe PCA. Analysis of bone quality on panoramic radiograph in osteoporosis research by fractal dimension. Appl Math (Irvine) 2016;7:375-386

3 Abramoff M, Magalhaes P, Ram SJ. Image processing with ImageJ. Biophoton Int 2004;11(7):36-42
4 Kavitha M, Khan M, Vijayalakshmi KR. Fractal dimension analysis in digital periapical radiographs: a diagnostic indicator of osteoporosis in post-menopausal women. J Indian Acad Oral Med Radiol 2017;29:84-89

5 Amer ME, Heo MS, Brooks SL, Benavides E. Anatomical variations of trabecular bone structure in intraoral radiographs using fractal and particles count analyses. Imaging Sci Dent 2012;42(1):5-12

6 Haire TJ, Hodgskinson R, Ganney PS, Langton CM. A comparison of porosity, fabric and fractal dimension as predictors of the Young's modulus of equine cancellous bone. Med Eng Phys 1998;20(8):588-593

7 White SC, Rudolph DJ. Alterations of the trabecular pattern of the jaws in patients with osteoporosis. Oral Surg Oral Med Oral Pathol Oral Radiol Endod 1999;88(5):628-635

8 Tosoni GM, Lurie AG, Cowan AE, Burleson JA. Pixel intensity and fractal analyses: detecting osteoporosis in perimenopausal and postmenopausal women by using digital panoramic images. Oral Surg Oral Med Oral Pathol Oral Radiol Endod 2006;102(2):235-241

9 Lee BD, White SC. Age and trabecular features of alveolar bone associated with osteoporosis. Oral Surg Oral Med Oral Pathol Oral Radiol Endod 2005;100(1):92-98

10 Southard TE, Southard KA, Jakobsen JR, Hillis SL, Najim CA. Fractal dimension in radiographic analysis of alveolar process bone. Oral Surg Oral Med Oral Pathol Oral Radiol Endod 1996;82(5):569-576

11 Pouilles JM, Tremollieres F, Todorovsky N, Ribot C. Precision and sensitivity of dual-energy $\mathrm{x}$-ray absorptiometry in spinal osteoporosis. J Bone Miner Res 1991;6(9):997-1002

12 Norton MR, Gamble C. Bone classification: an objective scale of bone density using the computerized tomography scan. Clin Oral Implants Res 2001;12(1):79-84

13 Lee DH, Ku Y, Rhyu IC, et al. A clinical study of alveolar bone quality using the fractal dimension and the implant stability quotient. J Periodontal Implant Sci 2010;40(1):19-24 